\title{
The Influence of Commercial Film-Forming Polymers on Reducing Salt Spray Injury in Evergreen Oak (Quercus ilex L.) and Laurel (Prunus laurocerasus L.)
}

\author{
Glynn C. Percival and Gillian A. Fraser
}

\begin{abstract}
A field trial was undertaken to determine the influence of four commercially available film-forming polymers (Bond [alkyl phenyl hydroxyl polyoxyethylene], Newman Crop Spray 11E ${ }^{\mathrm{TM}}$ [paraffinic oil], Nu-Film P [poly-1-p menthene], and Spray Gard [di-1-p menthene]) on reducing salt spray injury on two woody species, evergreen oak (Quercus ilex L.) and laurel (Prunus laurocerasus L.). Irrespective of species, the film-forming polymers Nu-Film-P and Spay Gard did not provide any significant degree of protection against salt spray damage irrespective of concentration (1\% or $2 \%)$ applied as measured by leaf chlorophyll concentrations, photosynthetic efficiency, visual leaf necrosis, foliar sodium and chloride content, and growth (height, leaf area). The film-forming polymer Newman Crop Spray 11E 1-week protection against salt spray injury. The film-forming polymer Bond provided a significant $(P<0.05)$ degree of protection against salt spray injury 3 months after application as manifest by higher leaf chlorophyll content, photosynthetic efficiency, height and leaf area, and lower visual leaf necrosis and foliar $\mathrm{Na}$ and $\mathrm{Cl}$ content compared with nontreated controls. In conclusion, results indicate that application of a suitable film-forming polymer can provide a significant degree of protection of up to 3 months against salt spray injury in evergreen oak and laurel. Results also indicate that when applied at $1 \%$ or $2 \%$ solutions, no problems associated with phytotoxicity and rapid degradation on the leaf surface exist.
\end{abstract}

Key Words. Antitranspirants; carotenoids; chlorophyll fluorescence; chlorophylls; English oak; laurel; leaf necrosis; photosystem II; sodium chloride; urban trees.

Deicing salts in the form of sodium chloride $(\mathrm{NaCl})$ are used widely throughout the United Kingdom to maintain roads free from ice and snow to ensure public safety (Ryan 2005; Zimmerman and Jull 2006). However, $\mathrm{NaCl}$ can be a major chemical pollutant in urban landscapes having a detrimental effect on roadside vegetation (Percival et al. 2003). Salt damage occurs by direct toxicity of the chloride ion resulting in a reduction in leaf chlorophyll concentration and concomitant photosynthetic performance, breakdown of leaf structure at the cellular level, necrosis, bud failure, and twig and branch dieback (Zhu 2001). Sodium tends to damage soil structure by competition with other cation exchange sites causing nutrient deficiency symptoms and increased soil $\mathrm{pH}$ (Gibbs and Palmer 1994). Deicing salts are estimated to be directly responsible for the deaths of $>700,000$ trees annually in Western Europe alone (Dobson 1991) and are particularly devastating to young spring growth that is unable to acclimate and therefore extremely susceptible. With increases in traffic volume and the expansion of road networks throughout the United Kingdom, the quantity of salt used for deicing operations has increased correspondingly. Consequently, there is a demand for salt-protectant compounds that are inexpensive, can be applied at relatively short notice, and require only small adjustments to existing management aftercare procedures (Percival and Barnes 2005).

Film-forming polymers and spray oils are widely used as spray adjuvants and antitranspirants within the agricultural and horticultural industries. When applied, they form a physical barrier across the leaf or twig surface and concomitantly form an insulating layer between the leaf/twig surface and atmosphere (Fuller et al. 2003). Such effects may protect against direct salt damage caused by coastal spray, rain, and/ or traffic splash of water contaminated with deicing salt. When tested by a number of researchers in the 1970s, most concluded that antitranspirant and antidessication-based filmforming polymers did not offer promise as a feasible salt amelioration technique either proving to be ineffective or directly phytotoxic to leaf tissue (Constantini and Rich 1973; Emmons et al. 1976; Sauer 1980). Developments in polymer technology, however, have led to the formulation of a range of new polymers that contain different active ingredients from the original products used in the earlier trials that are 
more stable and nontoxic to leaf tissue. Several studies have found these film-forming polymers to be effective in reducing salt and freezing damage to crop plants of economic significance (Fuller et al. 2003). Whether applications of these newly developed polymers can improve the salt tolerance of urban trees remains untested.

\section{MATERIALS AND METHODS}

\section{Plant Material and Experimental Design}

The experiment used 3-year-old, bare-rooted stock of evergreen oak (Quercus ilex L.) laurel (Prunus laurocerasus L.). Trees were planted directly into the ground by hand in early November 2002 and left to establish throughout the 2003 growing season (April through September). The trial site was located at the University of Reading Shinfield Experimental Site, Berkshire. Planting distances were $1 \times 1 \mathrm{~m}(3.3 \times 3.3 \mathrm{ft})$. Supplementary irrigation was applied during the 2003 growing season and trees were fertilized in early May 2003 by a broadcast application of granular N:P:K (29:7:9) fertilizer (Bartlett BOOST; The Doggett Corporation, Lebanon, NJ) applied at $40 \mathrm{~g} / \mathrm{m}^{2}\left(1.4 \mathrm{oz} / 11 \mathrm{ft}^{2}\right)$. The soil was a sandy loam containing $4 \%$ to $6 \%$ organic matter, $\mathrm{pH}$ of 6.2 ; available $\mathrm{P}$, $\mathrm{K}, \mathrm{Mg}, \mathrm{Na}$, and $\mathrm{Ca}$ were 52.0, 659.1, 175.2, 49.4, and 2188 $\mathrm{mg} / \mathrm{L}(0.0001,0.005,0.002,0.0001,0.02 \mathrm{oz} / \mathrm{gal})$, respectively, at the time treatments began (November 2003). Weeds were controlled chemically using glyphosate (Roundup; Green-Tech, Sweethills Park, Nun Monkton, York, U.K.) from the day of planting, November 2002, to the cessation of the experiment, March 2004. One week before the trial trees were inspected and only those rated with a leaf visual index of zero (no leaf necrosis or foliar discoloration observed) were used in the trial. The experimental design was a randomized complete block with five single tree replications per treatment. To keep the physiological age of the leaves comparable throughout the experiment, measurements of chlorophyll fluorescence and chlorophyll content (SPAD) were made only on fully expanded leaves present at the commencement of the trial. Before film-forming polymer sprays, polythene screens $1 \mathrm{~m}(3.3 \mathrm{ft})$ high were erected around each tree to prevent dispersal of sprays and possible cross-contact with other trees and the base of the tree was covered with a $0.5 \mathrm{~m} \times 0.5 \mathrm{~m}(1.65 \times 1.65 \mathrm{ft})$ polythene mulch to prevent percolation into the soil.

\section{Film-Forming Polymers}

Foliar sprays of the film-forming polymers Bond (active ingredient [a.i.] alkyl phenyl hydroxyl polyoxyehtylene), Newman Crop Spray 11E ${ }^{\mathrm{TM}}$ (a.i. paraffinic oil), Nu-Film P (a.i. poly-1-p menthene), and Spray Gard (a.i. di-1-p menthene) were applied until runoff using a hand sprayer at a concentration of $10(0.3)$ and $20 \mathrm{~mL}(0.6 \mathrm{fl} \mathrm{oz})$ per liter $(0.26 \mathrm{gal})$ of water. All film-forming polymers were obtained from United Agri Products Ltd., Alconbury Weston, Huntingdon, U.K.
Trees were sprayed on 1 November 2003 and 15 trees per polymer treatment were used.

\section{Salt Treatments}

To assess the longevity and durability of each polymer on the leaf surface at week 1 (7 November 2003), month 1 (7 December 2003), and month 3 (7 February 2004) after polymer application, trees were foliar-sprayed with a $60 \mathrm{~g}(2.1 \mathrm{oz})$ sodium chloride per liter $(0.26 \mathrm{gal})$ distilled water solution $(6 \% \mathrm{NaCl}$ solution) until runoff. Five different trees were sprayed with salt at each time interval.

\section{Chlorophyll Fluorescence}

Fluorescence values were obtained by placing leaves in darkness for $30 \mathrm{~min}$ and attaching light exclusion clips to the leaf of whole trees and chlorophyll fluorescence was measured using a HandyPEA portable fluorescence spectrometer (Hansatech Instruments Ltd., King's Lynn, U.K.). Measurements were recorded up to $1 \mathrm{sec}$ with a data acquisition rate of $10 \mu \mathrm{s}$ for the first $2 \mathrm{~ms}$ and of $1 \mathrm{~ms}$ thereafter. The fluorescence responses were induced by a red (peak at $660 \mathrm{~nm}$ ) light of $1500 \mu \mathrm{mol} / \mathrm{m}^{-2} / \mathrm{s}^{-1}$ photosynthetically active radiation intensity provided by an array of six red light-emitting diodes. A photosynthetic performance index (PI) based on an equation that combines the relationship of calculated relative number of reaction centers (RC) per energy absorbed (ABS) and then multiplied by two expressions describing the yields of light trapping $(\phi p o)$ and subsequent electron transport $\left(\Psi_{0}\right)$ i.e., $\mathrm{RC} / \mathrm{ABS} \times \phi \mathrm{po} /(1-\phi \mathrm{po}) \times \Psi_{0} /\left(1-\Psi_{0}\right.$; Clark et al. 1998, 2000) were used to quantify any effects on leaf tissue. PI values have been shown to be a highly sensitive measure of leaf photosynthetic activities as an indirect measure of plant vitality (Percival and Fraser 2001). PI values were automatically calculated by the HandyPEA. Measurements were made at day 14 after salt treatment at each time (week 1, months 1 and 3) interval.

\section{Chlorophyll Measurements}

A Minolta chlorophyll meter SPAD-502 (Spectrum Technologies, Inc., East Plainfield, IL, U.S.) was used. Chlorophyll was measured at the midpoint of the leaf next to the main leaf vein. Calibration was obtained by measurement of absorbance at 663 and $645 \mathrm{~nm}$ in a spectrophotometer (PU8800 Pye Unicam; Fisher Scientific UK Ltd., Loughborough, Leicestershire, U.K.) after extraction with $80 \% \mathrm{v} / \mathrm{v}$ aqueous acetone (regression equation $=5.80+0.057 \mathrm{x} ; \mathrm{r}^{2}$ adjusted $=0.82, P<0.01$ ) (Lichtenthaler and Wellburn 1983). Measurements were made at day 14 after salt treatment at each time (week 1, months 1 and 3 ) interval.

\section{Leaf Necrosis}

Leaf necrosis was assessed visually at day 14 after salt treatment. Each tree was rated on a 0 to 5 rating scale using a visual indexing technique and ratings on the scale: $0=$ no 
necrosis observed; $1=$ less than $5 \%$ of leaves affected and no aesthetic impact; $2=5 \%$ to $20 \%$ of leaves affected with some yellowing but little or no defoliation; $3=21 \%$ to $50 \%$ of leaves affected, significant defoliation and/or leaf yellowing; $4=51 \%$ to $80 \%$ of leaves affected, severe foliar discoloration; $5=81 \%$ to $100 \%$ of foliage affected with $90 \%$ to $100 \%$ defoliation. The individual ratings for each tree in each treatment were used for statistical analysis.

\section{Foliar Sodium and Chloride Analysis}

Leaf samples (six leaves per tree) were thoroughly washed and then dried in a convection oven at $85^{\circ} \mathrm{C}\left(185^{\circ} \mathrm{F}\right)$ for $48 \mathrm{hr}$ before grinding through a $0.5 \mathrm{~mm}(0.02$ in) cyclone mill (Retsch, Middlesborough, U.K.). Samples ( $0.5 \mathrm{~g}$ ) were placed into $150 \mathrm{~mL}(4.5 \mathrm{fl} \mathrm{oz})$ volumetric flasks and digested in 20 $\mathrm{mL}(0.6 \mathrm{fl} \mathrm{oz})$ of $7: 1$ nitric/perchloric acid. After cooling, the solutions were brought to volume with deionized water and analyzed by inductively coupled plasma-emission spectroscopy elemental analysis. Nutrient values were expressed as grams per kilogram dry weight. Measurements were made at day 14 after salt treatment at each time (week 1, months 1 and 3) interval.

\section{Plant Height and Leaf Area}

At the end of the 2004 growing season (23 September), trees were destructively harvested and height recorded by measuring the distance from the tip of the leading apical shoot to the soil surface. Leaf areas were quantified using a Delta-T area meter (Delta-T Devices, Ltd., Cambridge, U.K.).

\section{Data Analysis}

Statistical methods were as follows: before the analysis, data were examined to ensure normality and homogeneity of variance (Anderson-Darling test) was met to meet the assumptions for the analysis of variance. Data were then analyzed as a randomized complete block using an analysis of variance. Differences in levels of leaf necrosis severity, chlorophyll fluorescence, and SPAD readings from control values were determined and means were separated at the $P<0.05$ level of significance using the Genstat for Windows program.

\section{RESULTS AND DISCUSSION}

The influence of species $(\mathrm{S})$, polymer $(\mathrm{P})$, time of salt application (T), and salient interactions of these three factors on leaf necrosis, $\mathrm{PI}$, chlorophyll content, leaf $\mathrm{Na}$ and $\mathrm{Cl}$ content, height and leaf area singly and in combination are shown in Table 1.

During the study, none of the treated or control trees died as a result of salt spray. Salt damage was recorded on control trees as indicated by leaf necrosis ratings between 3.6 and 3.9 on foliar tissue of evergreen oak and laurel (Tables 2 and 3). Increased salt damage on controls was mirrored by reduced leaf PI and chlorophyll content, elevated leaf $\mathrm{Na}$ and $\mathrm{Cl}$ content, and reduced height and leaf area (Tables 2 and 3).

Irrespective of tree species the film-forming polymers $\mathrm{Nu}-$ Film-P and Spray Gard did not provide any significant degree of protection against salt-induced damage irrespective of concentration $(1 \%$ or $2 \%)$ applied. Chlorophyll concentrations, leaf PI values as a measure of photosynthetic efficiency, visual leaf necrosis (Tables 2 and 3), foliar $\mathrm{Na}$ and $\mathrm{Cl}$ (Tables 4 and 5), content, and growth (height, leaf area; Tables 6 and 7) did not significantly differ from control values with two exceptions: evergreen oak after application of Spray Gard at $2 \%$ where leaf necrosis values were significantly lower $(P<0.05)$ than controls at week 1 and laurel also after application of Spray Gard at $1 \%$ where leaf area values that were significantly higher $(P<0.05)$ at month 3 . Failure of these two film-forming polymers to provide any form of protection against salt damage is in agreement with other workers who also found poly-1-p menthene (Vapour Gard; Miller Chemical \& Fertilizer Corp., Hanover, PA, U.S.) and di-1-p menthene (Wilt-Pruf; Wilt-Pruf Products, Inc., Essex, CT, U.S.) -based products ineffective as potential ameliorants to reduce salt spray injury on woody plants (Emmons et al. 1976). Sauer (1980) also found several types of predominantly poly and di-1-p menthene-based film-forming polymers ineffective in protecting roadside plants from salt spray injury.

However, contrary to previous work (Constantini and Rich 1973; Emmons et al. 1976) phytotoxicity and enhanced leaf $\mathrm{Na}$ and $\mathrm{Cl}$ contents was not observed in the two test species

Table 1. $P$ values for growth and tree vitality post salt treatment.

\begin{tabular}{|c|c|c|c|c|c|c|c|}
\hline Factor & Chlorophyll content & PI & Leaf necrosis & Leaf $\mathrm{Na}$ content & Leaf $\mathrm{Cl}$ content & Height & Leaf area \\
\hline Species (S) & $<0.001$ & $<0.001$ & 0.004 & 0.222 & 0.216 & $<0.001$ & $<0.001$ \\
\hline Polymer (P) & $<0.001$ & $<0.001$ & $<0.001$ & $<0.001$ & $<0.001$ & $<0.001$ & $<0.001$ \\
\hline Time $(\mathrm{T})$ & $<0.001$ & $<0.001$ & 0.001 & $<0.001$ & $<0.001$ & $<0.001$ & 0.006 \\
\hline $\mathrm{S} * \mathrm{P}$ & 0.145 & $<0.001$ & $<0.010$ & 0.416 & 0.231 & 0.456 & 0.040 \\
\hline $\mathrm{S} * \mathrm{~T}$ & 0.026 & $<0.001$ & 0.166 & 0.866 & 0.467 & 0.342 & 0.011 \\
\hline $\mathrm{P} * \mathrm{~T}$ & $<0.001$ & $<0.001$ & $<0.001$ & $<0.001$ & $<0.001$ & 0.015 & 0.149 \\
\hline $\mathrm{S} * \mathrm{P} * \mathrm{~T}$ & 0.241 & $<0.001$ & 0.887 & 0.509 & 0.778 & 0.461 & 0.938 \\
\hline
\end{tabular}

$\mathrm{PI}=$ photosynthetic performance index; $\mathrm{Na}=$ sodium; $\mathrm{Cl}=$ chloride. $P<0.05$ significant at the $95 \%$ confidence level; $P<0.01$, significant at the $99 \%$ confidence level; $P<0.05$ not significant. 
Table 2. Influence of film-forming polymers on leaf photosynthetic efficiency (PI), chlorophyll concentration, and necrosis of evergreen oak under field conditions after applications of $6 \%$ salt $(\mathrm{NaCl})$ at week 1 and months 1 and 3 after polymer application.

\begin{tabular}{|c|c|c|c|c|c|c|c|c|c|}
\hline \multirow[b]{2}{*}{ Treatment } & \multicolumn{3}{|c|}{ Week 1} & \multicolumn{3}{|c|}{ Month 1} & \multicolumn{3}{|c|}{ Month 3} \\
\hline & PI & $\begin{array}{l}\text { Chlorophyll } \\
\text { concentration } \\
\text { (SPAD) }\end{array}$ & $\begin{array}{l}\text { Leaf } \\
\text { necrosis }\end{array}$ & PI & $\begin{array}{l}\text { Chlorophyll } \\
\text { concentration } \\
\text { (SPAD) }\end{array}$ & $\begin{array}{l}\text { Leaf } \\
\text { necrosis }\end{array}$ & PI & $\begin{array}{l}\text { Chlorophyll } \\
\text { concentration } \\
\text { (SPAD) }\end{array}$ & $\begin{array}{l}\text { Leaf } \\
\text { necrosi }\end{array}$ \\
\hline Control & 1.43 & 21.73 & 3.9 & 1.17 & 18.67 & 3.9 & 1.18 & 17.81 & 3.9 \\
\hline Nu Film P $1 \%$ & $1.61^{\mathrm{ns}}$ & $21.19^{\mathrm{ns}}$ & $3.1^{\mathrm{ns}}$ & $1.46^{\mathrm{ns}}$ & $20.78^{\text {ns }}$ & $3.6^{\mathrm{ns}}$ & $1.43^{\mathrm{ns}}$ & $19.51^{\mathrm{ns}}$ & $3.6^{\mathrm{ns}}$ \\
\hline Nu Film P $2 \%$ & $1.47^{\mathrm{ns}}$ & $20.93^{\text {ns }}$ & $4.5^{\mathrm{ns}}$ & $0.83^{\text {ns }}$ & $16.86^{\text {ns }}$ & $4.3^{\mathrm{ns}}$ & $1.38^{\mathrm{ns}}$ & $19.59^{\text {ns }}$ & $4.0^{\mathrm{ns}}$ \\
\hline Spray Gard $1 \%$ & $1.39^{\mathrm{ns}}$ & $22.24^{\mathrm{ns}}$ & $3.0^{\text {ns }}$ & $0.88^{\text {ns }}$ & $17.39^{\text {ns }}$ & $3.0^{\mathrm{ns}}$ & $0.83^{\mathrm{ns}}$ & $17.53^{\text {ns }}$ & $3.1^{\mathrm{ns}}$ \\
\hline Spray Gard $2 \%$ & $1.31^{\mathrm{ns}}$ & $20.67^{\mathrm{ns}}$ & $2.8^{z}$ & $0.81^{\mathrm{ns}}$ & $16.32^{\text {ns }}$ & $3.1^{\mathrm{ns}}$ & $1.18^{\mathrm{ns}}$ & $18.00^{\text {ns }}$ & $3.3^{\text {ns }}$ \\
\hline Bond $1 \%$ & $2.57^{\mathrm{z}}$ & $28.10^{\mathrm{z}}$ & $0.7^{z}$ & $2.44^{\mathrm{z}}$ & $26.15^{\mathrm{z}}$ & $0.9^{z}$ & $2.57^{\mathrm{z}}$ & $23.28^{\mathrm{z}}$ & $1.3^{\mathrm{z}}$ \\
\hline Bond $2 \%$ & $1.98^{\text {ns }}$ & $26.13^{z}$ & $1.1^{\mathrm{z}}$ & $1.71^{\mathrm{ns}}$ & $26.38^{z}$ & $1.4^{\mathrm{z}}$ & $1.95^{\mathrm{z}}$ & $22.30^{z}$ & $1.9^{\mathrm{z}}$ \\
\hline Crop Spray $1 \%$ & $1.99^{\mathrm{ns}}$ & $25.60^{\mathrm{z}}$ & $1.7^{\mathrm{z}}$ & $1.28^{\text {ns }}$ & $20.16^{\mathrm{ns}}$ & $2.4^{\mathrm{z}}$ & $1.36^{\mathrm{ns}}$ & $19.59^{\text {ns }}$ & $3.9^{\text {ns }}$ \\
\hline Crop Spray $2 \%$ & $2.06^{\mathrm{z}}$ & $26.02^{z}$ & $1.3^{\mathrm{z}}$ & $0.82^{\mathrm{ns}}$ & $15.14^{\mathrm{ns}}$ & $2.9^{\text {ns }}$ & $1.35^{\mathrm{ns}}$ & $20.04^{\mathrm{ns}}$ & $3.5^{\mathrm{ns}}$ \\
\hline LSD & 0.619 & 3.252 & 1.08 & 0.603 & 2.842 & 1.49 & 0.651 & 3.126 & 1.17 \\
\hline
\end{tabular}

All PI and chlorophyll concentration values mean of five trees, six leaves per tree. All necrosis values mean of five trees.

${ }^{\mathrm{z}}$ Significantly different from controls according to least significant difference (LSD) at $P<0.05$.

$\mathrm{ns}=$ not significant different from control value.

after application of poly-1-p menthene (Nu-Film-P) and di1-p menthene (Spray Gard) at the higher concentration of $2 \%$. Marked sensitivity between species in response to antitranspirant and antidessicant-based film-forming polymers has been shown elsewhere (Sauer 1980). Possibly the species used in this investigation was tolerant of the higher concentrations applied. Alternatively differing methodologies between this study and that of Constantini and Rich (1973) may account for the difference in results. In the case of Constantini and Rich (1973), the film-forming polymers were evaluated as therapeutic remedies, i.e., applied simultaneously with or after salt spray application to foliar tissue. In this investigation, film-forming polymers were evaluated for any possible preventive properties and as such applied to foliar tissue at least 1 week before salt spray application.

Newman Crop Spray 11E $\mathrm{E}^{\mathrm{TM}}$ is a paraffinic oil-based product primarily used to enhance the efficacy of herbicides such as glyphosate and 2,4-D in arable systems (Anonymous 2006a). In addition, spray oils are widely used as suffocants to control a range of insect pests such as aphids, leafhoppers, and scale (Anonymous 2006b). The influence of a spray oil product on reducing salt spray damage has not been investigated. Results

Table 3. Influence of film-forming polymers on leaf photosynthetic efficiency (PI), chlorophyll concentration, and necrosis of laurel under field conditions after applications of $6 \%$ salt $(\mathrm{NaCl})$ at week 1 and months 1 and 3 after polymer application.

\begin{tabular}{|c|c|c|c|c|c|c|c|c|c|}
\hline \multirow[b]{2}{*}{ Treatment } & \multicolumn{3}{|c|}{ Week 1} & \multicolumn{3}{|c|}{ Month 1} & \multicolumn{3}{|c|}{ Month 3} \\
\hline & PI & $\begin{array}{l}\text { Chlorophyll } \\
\text { concentration } \\
\text { (SPAD) }\end{array}$ & $\begin{array}{l}\text { Leaf } \\
\text { necrosis }\end{array}$ & PI & $\begin{array}{l}\text { Chlorophyll } \\
\text { concentration } \\
\text { (SPAD) }\end{array}$ & $\begin{array}{l}\text { Leaf } \\
\text { necrosis }\end{array}$ & PI & $\begin{array}{l}\text { Chlorophyll } \\
\text { concentration } \\
\text { (SPAD) }\end{array}$ & $\begin{array}{l}\text { Leaf } \\
\text { necrosis }\end{array}$ \\
\hline Control & 3.50 & 28.2 & 3.8 & 2.35 & 26.7 & 3.6 & 2.60 & 24.0 & 3.8 \\
\hline Nu Film P $1 \%$ & $3.33^{\mathrm{ns}}$ & $30.0^{\mathrm{ns}}$ & $4.3^{\mathrm{ns}}$ & $2.21^{\mathrm{ns}}$ & $24.2^{\mathrm{ns}}$ & $3.5^{\mathrm{ns}}$ & $2.47^{\mathrm{ns}}$ & $25.2^{\mathrm{ns}}$ & $3.4^{\mathrm{ns}}$ \\
\hline Nu Film P $2 \%$ & $3.94^{\mathrm{ns}}$ & $28.6^{\mathrm{ns}}$ & $3.5^{\mathrm{ns}}$ & $2.06^{\mathrm{ns}}$ & $24.7^{\mathrm{ns}}$ & $3.8^{\mathrm{ns}}$ & $2.90^{\mathrm{ns}}$ & $22.6^{\mathrm{ns}}$ & $3.6^{\mathrm{ns}}$ \\
\hline Spray Gard $1 \%$ & $4.01^{\mathrm{ns}}$ & $28.9^{\mathrm{ns}}$ & $4.3^{\mathrm{ns}}$ & $2.36^{\mathrm{ns}}$ & $23.8^{\mathrm{ns}}$ & $4.1^{\mathrm{ns}}$ & $2.24^{\mathrm{ns}}$ & $22.1^{\mathrm{ns}}$ & $3.9^{\mathrm{ns}}$ \\
\hline Spray Gard $2 \%$ & $1.85^{\mathrm{ns}}$ & $23.8^{\mathrm{ns}}$ & $3.9^{\mathrm{ns}}$ & $1.26^{\mathrm{ns}}$ & $20.6^{\mathrm{ns}}$ & $3.9^{\mathrm{ns}}$ & $1.71^{\mathrm{ns}}$ & $20.5^{\mathrm{ns}}$ & $3.7^{\mathrm{ns}}$ \\
\hline Bond $1 \%$ & $7.96^{\mathrm{z}}$ & $34.2^{\mathrm{z}}$ & $1.3^{\mathrm{z}}$ & $6.78^{\mathrm{z}}$ & $29.1^{\mathrm{ns}}$ & $1.8^{\mathrm{z}}$ & $4.48^{\mathrm{z}}$ & $28.0^{\mathrm{z}}$ & $2.4^{\mathrm{z}}$ \\
\hline Bond $2 \%$ & $5.53^{\mathrm{z}}$ & $32.9^{\mathrm{z}}$ & $1.6^{\mathrm{z}}$ & $5.44^{z}$ & $30.1^{\mathrm{z}}$ & $1.5^{\mathrm{z}}$ & $5.04^{\mathrm{z}}$ & $31.4^{z}$ & $1.9^{\mathrm{z}}$ \\
\hline Crop Spray $1 \%$ & $6.88^{\mathrm{z}}$ & $34.5^{\mathrm{z}}$ & $2.3^{\mathrm{z}}$ & $3.12^{\mathrm{ns}}$ & $25.9^{\mathrm{ns}}$ & $3.7^{\mathrm{ns}}$ & $2.60^{\mathrm{ns}}$ & $23.0^{\mathrm{ns}}$ & $4.2^{\mathrm{ns}}$ \\
\hline Crop Spray 2\% & $6.52^{\mathrm{z}}$ & $34.5^{z}$ & $1.5^{\mathrm{z}}$ & $3.38^{\mathrm{z}}$ & $23.6^{\mathrm{ns}}$ & $3.6^{\mathrm{ns}}$ & $2.36^{\mathrm{ns}}$ & $22.8^{\mathrm{ns}}$ & $3.3^{\mathrm{ns}}$ \\
\hline LSD & 1.380 & 2.217 & 1.10 & 0.837 & 2.877 & 1.05 & 0.812 & 3.018 & 0.79 \\
\hline
\end{tabular}

All PI and chlorophyll concentration values mean of five trees, six leaves per tree. All necrosis values mean of five trees.

${ }^{\mathrm{z}}$ Significantly different from controls according to least significant difference (LSD) at $P<0.05$.

ns $=$ not significant different from control value. 
Table 4. Influence of film-forming polymers on sodium chloride concentrations in leaf tissue of evergreen oak under field conditions after applications of $6 \%$ salt $(\mathrm{NaCl})$ at week 1 and months 1 and 3 after polymer application.

\begin{tabular}{|c|c|c|c|c|c|c|}
\hline \multirow[b]{2}{*}{ Treatment } & \multicolumn{2}{|c|}{ Week 1} & \multicolumn{2}{|c|}{ Month 1} & \multicolumn{2}{|c|}{ Month 3} \\
\hline & Leaf $\mathrm{Na}$ content & Leaf $\mathrm{Cl}$ content & Leaf $\mathrm{Na}$ content & Leaf $\mathrm{Cl}$ content & Leaf $\mathrm{Na}$ content & Leaf $\mathrm{Cl}$ content \\
\hline Control & 40.2 & 51.4 & 38.1 & 54.7 & 38.3 & 52.2 \\
\hline Nu Film P $1 \%$ & $36.6^{\mathrm{ns}}$ & $48.8^{\mathrm{ns}}$ & $41.0^{\mathrm{ns}}$ & $44.4^{\mathrm{ns}}$ & $39.4^{\mathrm{ns}}$ & $48.3^{\mathrm{ns}}$ \\
\hline $\mathrm{Nu}$ Film P 2\% & $40.5^{\mathrm{ns}}$ & $54.2^{\mathrm{ns}}$ & $43.3^{\mathrm{ns}}$ & $50.9^{\mathrm{ns}}$ & $36.8^{\mathrm{ns}}$ & $48.5^{\mathrm{ns}}$ \\
\hline Spray Gard $1 \%$ & $37.6^{\mathrm{ns}}$ & $45.7^{\mathrm{ns}}$ & $38.0^{\mathrm{ns}}$ & $50.8^{\mathrm{ns}}$ & $40.0^{\text {ns }}$ & $53.8^{\mathrm{ns}}$ \\
\hline Spray Gard $2 \%$ & $39.1^{\mathrm{ns}}$ & $51.0^{\mathrm{ns}}$ & $35.0^{\mathrm{ns}}$ & $52.2^{\mathrm{ns}}$ & $33.7^{\mathrm{ns}}$ & $45.1^{\mathrm{ns}}$ \\
\hline Bond $1 \%$ & $21.4^{\mathrm{z}}$ & $20.3^{z}$ & $15.9^{\mathrm{z}}$ & $23.1^{\mathrm{z}}$ & $19.0^{\mathrm{z}}$ & $29.9^{\mathrm{z}}$ \\
\hline Bond $2 \%$ & $19.3^{z}$ & $25.4^{\mathrm{z}}$ & $17.0^{\mathrm{z}}$ & $22.2^{\mathrm{z}}$ & $22.3^{\mathrm{z}}$ & $31.7^{\mathrm{z}}$ \\
\hline Crop Spray 1\% & $17.1^{\mathrm{z}}$ & $23.2^{\mathrm{z}}$ & $34.7^{\mathrm{ns}}$ & $47.4^{\mathrm{ns}}$ & $40.9^{\text {ns }}$ & $51.1^{\mathrm{ns}}$ \\
\hline Crop Spray $2 \%$ & $16.8^{\mathrm{z}}$ & $20.2^{\mathrm{z}}$ & $37.6^{\mathrm{ns}}$ & $50.2^{\mathrm{ns}}$ & $45.2^{\mathrm{ns}}$ & $53.5^{\mathrm{ns}}$ \\
\hline LSD & 11.92 & 8.94 & 6.75 & 12.43 & 7.09 & 9.29 \\
\hline
\end{tabular}

Sodium and chloride concentration (g/kg dry weight). All values mean of five trees, six leaves per tree.

${ }^{\mathrm{z}}$ Significantly different from controls according to least significant difference (LSD) at $P<0.05$.

ns $=$ not significant different from control value.

of this investigation show that application of a paraffinic oil-based film-forming polymer provided a significant $(P<$ 0.05 ) degree of protection against foliar applied salt 1 week after application. Foliar leaf necrosis of evergreen oak and laurel was reduced by $40 \%$ to $70 \%$ compared with control trees (Tables 2 and 3). Such a reduction in leaf necrosis was significant from controls at $P<0.05$. In addition, photosynthetic leaf efficiency (PI) and leaf chlorophyll content were $\approx 28 \%$ to $49 \%$ and $18 \%$ to $20 \%$ higher, respectively (Tables 2 and 3). Likewise, reduced foliar $\mathrm{Na}(49 \%$ to $55 \%)$ and $\mathrm{Cl}$ $(50 \%$ to $60 \%)$ was recorded in both evergreen oak and laurel after foliar applications of salt 1 week after spray oil application compared with controls (Tables 4 and 5). Effects on growth varied between species. In the case of evergreen oak, height and leaf area were significantly higher $(P<0.05)$ than controls (Table 6). In the case of laurel, no significant effects were recorded with one exception: significantly $(P<0.05)$ increased leaf area after application at $2 \%$ Newman Crop Spray 11E $\mathrm{E}^{\mathrm{TM}}$ (Table 7). No significant effects on alleviating injury (leaf necrosis, PI, chlorophyll content), foliar $\mathrm{Na}$ and $\mathrm{Cl}$ content, and growth (height, leaf area) were recorded after salt application at months 1 and 3 after the application of spray oil with one exception: leaf necrosis in evergreen oak at month 1 in which values were significantly $(P<0.05)$ lower than controls. Such a response indicates only a short-term, i.e., $\leq 1$ week, protective influence of a paraffinic oil-based film-forming polymer against foliar salt-induced damage. Such a short-term response is undesirable for professionals involved in urban tree care because repeat visits for spray applications would involve higher labor costs and adjustments to standard aftercare management procedures. Ideally a "one off" long-term treatment to provide protection during

Table 5. Influence of film-forming polymers on sodium chloride concentrations in leaf tissue of laurel under field conditions after applications of $6 \%$ salt $(\mathrm{NaCl})$ at week 1 and months 1 and 3 after polymer application.

\begin{tabular}{|c|c|c|c|c|c|c|}
\hline \multirow[b]{2}{*}{ Treatment } & \multicolumn{2}{|c|}{ Week 1} & \multicolumn{2}{|c|}{ Month 1} & \multicolumn{2}{|c|}{ Month 3} \\
\hline & Leaf $\mathrm{Na}$ content & Leaf $\mathrm{Cl}$ content & Leaf $\mathrm{Na}$ content & Leaf $\mathrm{Cl}$ content & Leaf $\mathrm{Na}$ content & Leaf $\mathrm{Cl}$ content \\
\hline Control & 40.0 & 42.8 & 42.4 & 53.7 & 43.3 & 56.2 \\
\hline Nu Film P $1 \%$ & $36.8^{\mathrm{ns}}$ & $52.4^{\mathrm{ns}}$ & $40.3^{\mathrm{ns}}$ & $47.4^{\mathrm{ns}}$ & $39.0^{\mathrm{ns}}$ & $58.2^{\mathrm{ns}}$ \\
\hline Nu Film P 2\% & $42.0^{\mathrm{ns}}$ & $49.6^{\mathrm{ns}}$ & $39.4^{\mathrm{ns}}$ & $54.7^{\mathrm{ns}}$ & $41.8^{\mathrm{ns}}$ & $52.1^{\mathrm{ns}}$ \\
\hline Spray Gard 1\% & $38.9^{\mathrm{ns}}$ & $52.4^{\mathrm{ns}}$ & $39.6^{\mathrm{ns}}$ & $51.9^{\mathrm{ns}}$ & $45.1^{\mathrm{ns}}$ & $57.6^{\mathrm{ns}}$ \\
\hline Spray Gard 2\% & $41.0^{\mathrm{ns}}$ & $50.8^{\mathrm{ns}}$ & $43.6^{\mathrm{ns}}$ & $46.1^{\mathrm{ns}}$ & $37.8^{\mathrm{ns}}$ & $45.9^{\mathrm{ns}}$ \\
\hline Bond 1\% & $16.3^{\mathrm{z}}$ & $20.4^{\mathrm{z}}$ & $18.9^{\mathrm{z}}$ & $21.6^{\mathrm{z}}$ & $16.8^{\mathrm{z}}$ & $29.8^{z}$ \\
\hline Bond 2\% & $18.8^{\mathrm{z}}$ & $22.1^{\mathrm{z}}$ & $14.2^{\mathrm{z}}$ & $27.1^{\mathrm{z}}$ & $20.3^{\mathrm{z}}$ & $25.3^{\mathrm{z}}$ \\
\hline Crop Spray $1 \%$ & $19.7^{\mathrm{z}}$ & $23.2^{\mathrm{z}}$ & $40.5^{\mathrm{ns}}$ & $53.8^{\mathrm{ns}}$ & $38.7^{\mathrm{ns}}$ & $62.3^{\mathrm{ns}}$ \\
\hline Crop Spray 2\% & $23.9^{\mathrm{z}}$ & $28.9^{\mathrm{z}}$ & $38.1^{\mathrm{ns}}$ & $49.9^{\mathrm{ns}}$ & $35.3^{\mathrm{ns}}$ & $53.0^{\mathrm{ns}}$ \\
\hline LSD & 10.29 & 13.79 & 7.39 & 10.11 & 8.10 & 15.54 \\
\hline
\end{tabular}

Sodium and chloride concentration ( $\mathrm{g} / \mathrm{kg}$ dry weight). All values mean of five trees, six leaves per tree.

${ }^{\mathrm{z}}$ Significantly different from controls according to least significant difference (LSD) at $P<0.05$.

${ }^{\mathrm{ns}}=$ not significant different from control value. 
Table 6. Influence of film-forming polymers on height and leaf area of evergreen oak under field conditions after applications of $6 \%$ salt $(\mathrm{NaCl})$ at week 1 and months 1 and 3 after polymer application.

\begin{tabular}{|c|c|c|c|c|c|c|}
\hline \multirow[b]{2}{*}{ Treatment } & \multicolumn{2}{|r|}{ Week 1} & \multicolumn{2}{|r|}{ Month 1} & \multicolumn{2}{|r|}{ Month 3} \\
\hline & Height (m) & Leaf area $\left(\mathrm{cm}^{2}\right) \times 10^{2}$ & Height & Leaf area $\left(\mathrm{cm}^{2}\right) \times 10^{2}$ & Height & Leaf area $\left(\mathrm{cm}^{2}\right) \times 10^{2}$ \\
\hline Control & 1.00 & 21.9 & 0.95 & 21.7 & 1.06 & 23.1 \\
\hline Nu Film P $1 \%$ & $0.98^{\mathrm{ns}}$ & $24.0^{\mathrm{ns}}$ & $0.96^{\mathrm{ns}}$ & $23.1^{\mathrm{ns}}$ & $0.89^{\text {ns }}$ & $22.0^{\mathrm{ns}}$ \\
\hline Nu Film P 2\% & $1.03^{\mathrm{ns}}$ & $23.7^{\mathrm{ns}}$ & $0.94^{\mathrm{ns}}$ & $20.6^{\mathrm{ns}}$ & $1.05^{\mathrm{ns}}$ & $19.3^{\text {ns }}$ \\
\hline Spray Gard $1 \%$ & $1.08^{\mathrm{ns}}$ & $22.6^{\mathrm{ns}}$ & $1.04^{\mathrm{ns}}$ & $21.6^{\mathrm{ns}}$ & $0.97^{\mathrm{ns}}$ & $21.9^{\mathrm{ns}}$ \\
\hline Spray Gard $2 \%$ & $0.99^{\mathrm{ns}}$ & $21.5^{\mathrm{ns}}$ & $0.99^{\mathrm{ns}}$ & $22.3^{\text {ns }}$ & $1.04^{\mathrm{ns}}$ & $22.3^{\text {ns }}$ \\
\hline Bond $1 \%$ & $1.30^{\mathrm{z}}$ & $31.1^{\mathrm{z}}$ & $1.36^{\mathrm{z}}$ & $29.2^{\mathrm{z}}$ & $1.14^{\mathrm{ns}}$ & $26.5^{\mathrm{ns}}$ \\
\hline Bond $2 \%$ & $1.23^{\mathrm{z}}$ & $30.8^{z}$ & $1.23^{\mathrm{z}}$ & $28.9^{\mathrm{z}}$ & $1.19^{\mathrm{ns}}$ & $28.0^{\text {ns }}$ \\
\hline Crop Spray 1\% & $1.27^{\mathrm{z}}$ & $30.4^{\mathrm{z}}$ & $0.98^{\mathrm{ns}}$ & $25.1^{\mathrm{ns}}$ & $0.96^{\mathrm{ns}}$ & $21.3^{\mathrm{ns}}$ \\
\hline Crop Spray 2\% & $1.24^{\mathrm{z}}$ & $27.9^{\mathrm{z}}$ & $1.00^{\mathrm{ns}}$ & $21.4^{\mathrm{ns}}$ & $1.04^{\mathrm{ns}}$ & $18.7^{\mathrm{ns}}$ \\
\hline LSD & 0.166 & 5.29 & 0.170 & 4.48 & 0.164 & 5.40 \\
\hline
\end{tabular}

All values mean of five trees.

${ }^{\mathrm{z}}$ Significantly different from controls according to least significant difference (LSD) at $P<0.05$.

ns $=$ not significant different from control value.

the winter months when deicing salts are generally applied would be preferable.

Irrespective of concentration applied only the film-forming polymer Bond (alkyl phenyl hydroxyl polyoxyethylene) provided a significant $(P<0.05)$ degree of protection against salt-induced damage at week 1 and months 1 and 3 after application. At month 3, foliar leaf necrosis of both test species was reduced by $58 \%$ to $82 \%$ compared with nonpolymertreated controls, whereas leaf PI and chlorophyll content were $\approx 28 \%$ to $44 \%$ and $19 \%$ to $24 \%$ higher, respectively (Tables 2 and 3). Likewise, a significantly $(P<0.05)$ reduced foliar $\mathrm{Na}$ (39\% to $61 \%$ ) and $\mathrm{Cl}(38 \%$ to $45 \%)$ was recorded in both evergreen oak and laurel (Tables 4 and 5). In all cases, height and leaf area were higher $(P<0.05)$ in Bond-treated plants compared to controls, if not significantly so in all instances, at the cessation of the experiment (Tables 6 and 7). Such positive responses indicate that application of an alkyl phenyl hydroxyl polyoxyethylene-based film-forming polymer provides a significant degree of protection $\geq 3$ months against salt spray injury. One important property of film-forming polymers is their ability to stretch during leaf expansion. Although not investigated in this experiment, greater stretching properties of Bond over Nu-Film-P and Spray Gard might as a result protect leaf surfaces for a longer time period, and in addition newly expanded leaf tissue, accounting for the marked degree of protection recorded (Sutherland and Walters 2002). Likewise, both Nu-Film-P and Spray Gard have been used for many years primarily as antitranspirants to decrease water loss and wilting of commercially important forestry transplants as well as extend pesticide efficacy. The recent generation of film-forming polymers such as Bond contains different active ingredients that results of this inves-

Table 7. Influence of film-forming polymers on height and leaf area of laurel under field conditions after applications of $6 \%$ salt $(\mathrm{NaCl})$ at week 1 and months 1 and 3 after polymer application.

\begin{tabular}{|c|c|c|c|c|c|c|}
\hline \multirow[b]{2}{*}{ Treatment } & \multicolumn{2}{|r|}{ Week 1} & \multicolumn{2}{|r|}{ Month 1} & \multicolumn{2}{|r|}{ Month 3} \\
\hline & Height & Leaf area $\left(\mathrm{cm}^{2}\right) \times 10^{2}$ & Height & Leaf area $\left(\mathrm{cm}^{2}\right) \times 10^{2}$ & Height & Leaf area $\left(\mathrm{cm}^{2}\right) \times 10^{2}$ \\
\hline Control & 0.69 & 14.6 & 0.73 & 16.3 & 0.61 & 14.6 \\
\hline Nu Film P $1 \%$ & $0.63^{\mathrm{ns}}$ & $17.9^{\text {ns }}$ & $0.62^{\mathrm{ns}}$ & $17.5^{\mathrm{ns}}$ & $0.65^{\mathrm{ns}}$ & $17.9^{\text {ns }}$ \\
\hline Nu Film P 2\% & $0.66^{\mathrm{ns}}$ & $17.1^{\mathrm{ns}}$ & $0.64^{\mathrm{ns}}$ & $18.1^{\mathrm{ns}}$ & $0.63^{\mathrm{ns}}$ & $17.2^{\text {ns }}$ \\
\hline Spray Gard $1 \%$ & $0.62^{\mathrm{ns}}$ & $16.5^{\mathrm{ns}}$ & $0.64^{\mathrm{ns}}$ & $17.8^{\mathrm{ns}}$ & $0.57^{\mathrm{ns}}$ & $18.6^{\mathrm{z}}$ \\
\hline Spray Gard 2\% & $0.59^{\mathrm{ns}}$ & $17.8^{\mathrm{ns}}$ & $0.60^{\text {ns }}$ & $17.7^{\mathrm{ns}}$ & $0.68^{\mathrm{ns}}$ & $17.1^{\mathrm{ns}}$ \\
\hline Bond $1 \%$ & $0.88^{\mathrm{z}}$ & $21.2^{\mathrm{z}}$ & $0.76^{\mathrm{ns}}$ & $22.5^{z}$ & $0.77^{\mathrm{z}}$ & $21.0^{\mathrm{z}}$ \\
\hline Bond $2 \%$ & $0.76^{\mathrm{ns}}$ & $18.8^{\mathrm{ns}}$ & $0.81^{\mathrm{ns}}$ & $20.3^{\mathrm{ns}}$ & $0.84^{\mathrm{z}}$ & $20.2^{z}$ \\
\hline Crop Spray $1 \%$ & $0.72^{\mathrm{ns}}$ & $19.0^{\mathrm{ns}}$ & $0.63^{\text {ns }}$ & $16.6^{\mathrm{ns}}$ & $0.64^{\mathrm{ns}}$ & $17.3^{\mathrm{ns}}$ \\
\hline Crop Spray $2 \%$ & $0.79^{\text {ns }}$ & $21.5^{\mathrm{z}}$ & $0.65^{\mathrm{ns}}$ & $18.3^{\text {ns }}$ & $0.67^{\mathrm{ns}}$ & $18.5^{\mathrm{z}}$ \\
\hline LSD & 0.180 & 4.41 & 0.127 & 5.10 & 0.116 & 3.14 \\
\hline
\end{tabular}

All values mean of five trees.

${ }^{\mathrm{z}}$ Significantly different from controls according to least significant difference (LSD) at $P<0.05$.

ns $=$ not significant different from control value. 
tigation indicate are more stable and durable under outdoor weather conditions accounting for the longer degree of protection recorded (Sutherland and Walters 2002).

In all cases in which a significant degree of protection was conferred, a concomitant lower internal leaf $\mathrm{Na}$ and $\mathrm{Cl}$ content was also recorded. Such a response indicates that the effective film-forming polymers used in this study formed a physical barrier/protective coating across the leaf surface that prevented direct entry of $\mathrm{Na}$ and $\mathrm{Cl}$ ions into the leaf tissue. Natural precipitation during the experimental period may then have washed off and/or diluted $\mathrm{Na}$ and $\mathrm{Cl}$ ions to a concentration nonharmful to foliar tissue. Prevention of $\mathrm{Na}$ and $\mathrm{Cl}$ ions into leaf tissue would prove an important factor in reducing salt damage (delayed budbreak, reduced leaf size, leaf yellowing, and necrosis) because salt injury has been correlated with the accumulation of $\mathrm{Na}$ and $\mathrm{Cl}$ ions in plant tissue (Sucoff et al. 1976).

Irrespective of species, there was no marked difference in the level of protection conferred when Bond and Newman Crop Spray $11 \mathrm{E}^{\mathrm{TM}}$ were applied at $1 \%$ and $2 \%$. Such knowledge may prove useful to those involved in urban tree care in which the cost:benefit ratio involved with deicing salt protection needs to be considered.

In conclusion, results indicate that application of a suitable film-forming polymer can provide a significant degree of protection of up to 3 months against salt spray injury. Results also indicate that when applied, no problems associated with phytotoxicity and rapid degradation on the leaf surface exist. Improved hardiness against salt damage by the application of film-forming polymers ensures greater survival and enhanced aesthetics of trees located within urban landscapes during the winter months, in turn reducing potential labor and replacement costs. Importantly, film-forming polymers are commercially available, inexpensive, and, according to United Kingdom pesticide regulations, are classified as biologically inert meaning their toxicity to humans is negligible (Anonymous 2006b).

Acknowledgment. The authors are grateful for funding from the TREE Fund (John Z. Duling).

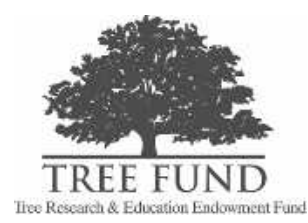

\section{LITERATURE CITED}

Anonymous. 2006a. De'Sangosse Agrochemical Product Manual. De'Sangosse Ltd., Swaffham Bulbeck, Cambridge, U.K.

. 2006b. The U.K. Pesticide Guide. British Crop Protection Council. Oxford, Cabi Publishing.
Clark, A.J., W. Landolt, J. Bucher, and R.J. Strasser. 1998. The response of Fagus sylvatica to elevated $\mathrm{CO}_{2}$ and ozone probed by the JIP-test based on the chlorophyll fluorescence rise: OJIP, pp. 283-286. In Responses of Plant Metabolism to Air Pollution and Global Change. De Kok, J.L., and Stulen, I., Eds. Leiden, The Netherlands, Brackhuys Publishers, Leiden.

- 2000. Beech (Fagus sylvatica L.) response to ozone exposure assessed with a chlorophyll a fluorescence performance index. Environmental Pollution (Barking, Essex: 1987) 109:501-507.

Constantini, A., and A.E. Rich. 1973. Comparison of salt injury to four species of coniferous tree seedlings when salt was applied to the potting medium and to the needles with and without an antitranspirants. Phytopathology 63: 200.

Dobson, M.C. 1991. De-icing salt damage to trees and shrubs. Forestry Commission Bulletin 101.

Emmons, A., A. Wood, and E. Sucoff. 1976. Antidesiccant sprays and damage from deicing salts. Minnesota Forestry Research Note 258.

Fuller, M.P., F. Hamed, M. Wisniewski, and D.M. Glenn. 2003. Protection of plants from frost using hydrophobic particle film and acrylic polymer. Annals of Applied Biology 143:93-97.

Gibbs, J.N., and C.A. Palmer. 1994. A survey of damage to roadside trees in London caused by the application of de-icing salt during the 1990/91 winter. Journal of Arboriculture 18:321-343.

Lichtenthaler, H.K., and A.R. Wellburn. 1983. Determinations of total carotenoids and chlorophylls a and b of leaf extracts in different solvents. Biochemical Society Transactions 11:591-593.

Percival, G.C., and S. Barnes. 2005. The influence of calcium fertilisation on the freezing and salinity tolerance of two urban tree species. Journal of Arboriculture 31:10-21.

Percival, G.C., and G.A. Fraser. 2001. Measurement of the salinity and freezing tolerance of Crataegus genotypes using chlorophyll fluorescence. Journal of Arboriculture 27:233-245.

Percival, G.C., G.A. Fraser, and G. Oxenham. 2003. Foliar salt tolerance of Acer genotypes using chlorophyll fluorescence. Journal of Arboriculture 29:61-66.

Ryan, J. 2005. Salt damage to trees. Essential ARB. 16: $11-12$.

Sauer, G. 1980. Experiments on protecting aerial parts of woody plants against direct effect of de-icing salt. Zeitschrift für Vegetation stechnik im Landschafts-und Sportsättenbau. 3:86-91.

Sucoff, E., S.G. Hong, and A. Wood. 1976. $\mathrm{NaCl}$ and twig dieback along highways and cold hardiness of highway versus garden twigs. Canadian Journal of Botany 54: $2268-2274$ 
Sutherland, F., and D.R. Walters. 2002. Effect of film forming polymers on infection of barley with the powdery mildew fungus, Bulmeria graminis f. sp. hordei. European Journal of Plant Pathology 108:385-389.

Zhu, J.K. 2001. Plant salt tolerance. Trends in Plant Science 6:66-71.

Zimmerman, E.M., and L.G. Jull. 2006. Sodium chloride injury on buds of Acer platanoides, tilia cordata and Viburnum lantana. Journal of Arboriculture 32:45-53.

\section{Glynn C. Percival (corresponding author)}

Plant Physiologist/Technical Support Specialist

R.A. Bartlett Tree Research Laboratory, Europe

The University of Reading

2 Earley Gate, Whiteknights

Reading, RG6 6AU, U.K.

gpercival@BartlettUK.com

\section{Gillian A Fraser \\ Research Lecturer \\ Centre for Excellence in Teaching and Learning \\ School of Agriculture, Policy and Development \\ Earley Gate \\ University of Reading \\ Reading, Berkshire \\ RG6 6AR, U.K.}

Résumé. Un essai sur le terrain a été mené afin d'évaluer l'influence de quatre films de polymères disponibles sur le marché commercial - Bond (alkyl phenyl hydroxyl polyoxyehtylene), Newman Crop Spray 11E $\mathrm{E}^{\mathrm{TM}}$ (huile de paraffine), Nu-Film P (poly-1-p menthene), Spray Gard (di-1-p menthene) - pour réduire les dommages causés par les embruns de sel sur deux espèces ligneuses, le Quercus ilex et le Prunus laurocerasus. En dépit de l'espèce, les films formés par le Nu-Film P et le Spray Gard ne fournissaient pas de degré significatif de protection contre les dommages par les embruns salés, en dépit de la concentration en sel appliquée (1 ou $2 \%$ ), et ce en fonction des mesures de concentration en chlorophylle foliaire, de l'efficacité photosynthétique, des nécroses foliaires visibles, du contenu en $\mathrm{Na}$ et en $\mathrm{Cl}$, et de la croissance (hauteur, surface foliaire). Le film formé par le polymère Newman Crop Spray $11 \mathrm{E}^{\mathrm{TM}}$ a fourni une protection durant une semaine seulement contre les dommages par les embruns salés. Le film produit par le Bond a donné un degré significatif de protection $(P<0,05)$ de protection contre les dommages par les embruns salés durant trois mois après son application, le tout se manifestant par un plus haut contenu en chlorophylle foliaire, en efficacité photosynthétique, en hauteur et en surface foliaire ainsi qu'un plus faible degré de nécroses foliaires visibles et de contenu foliaire en $\mathrm{Na}$ et $\mathrm{Cl}$, et ce en se comparant au groupe témoin. En conclusion, ces résultats in- diquent qu'une application d'un film adéquat de polymères peut fournir un degré de protection significatif jusqu'à trois mois contre les dommages par le embruns salés chez le $Q$. ilex et le $P$. laurocerasus. Les résultats indiquent aussi que lorsque le sel est appliqué à des taux de 1 ou $2 \%$ en solution, aucun problème associé de phytotoxicité et de rapide dégradation de la surface foliaire existe.

Zusammenfassung. Es wurde ein Feldversuch unternommen, um den Einfluss von vier kommerziell erhältlichen film-produzierenden Polymeren beider Reduzierung von Schäden durch Salz-Aerosole an zwei Baumarten, Immergrüne Eiche und Kirschlorbeer, zu bestimmen. Unabhängig von der Art lieferten die beiden Polymere „NuFilm-P“ und „Spray-Gard“ keinen signifikanten Schutz gegen Salzschäden, unabhängig von der Konzentration (1 oder $2 \%$ ), angewendet und gemessen durch Blattchlorophyll-Konzentration, Photosynthese-Leistung, sichtbare Blattnekrosen, NaCl-Gehalt der Blätter und Wachstum. Der filmbildende Polymer „Newman Crop Spray 11E" lieferte nur eine Woche Schutz gegen Salzspray. Der filmbildende Polymer „Bond“ lieferte einen signifikanten Schutz gegen Salzspray für drei Monate nach der Applikation, messbar durch höhere Blattchlorophyll-Konzentration, PhotosyntheseLeistung, weniger sichtbare Blattnekrosen und $\mathrm{NaCl}$-Gehalt der Blätter im Vergleich zu den unbehandelten Kontrollen. In der Zusammenfassung zeigten die Ergebnisse, dass die Applikation von geeigneten filmbildenden Polymeren einen deutlichen Schutz für bis zu drei Monaten gegen Salzspray bei Immergrünen Eichen und Kirschlorbeer liefern. Die Ergebnisse zeigen auch, dass, wenn 1 oder 2 \%ige Lösungen appliziert werden, keine Probleme mit Phytotoxizität und schneller Degradation auf der Blattoberfläche existieren.

Resumen. Se llevó a cabo un ensayo de campo para determinar la influencia de cuatro polímeros formadores de películas, comercialmente disponibles: (Bond (alkyl phenyl hydroxyl polyoxyehtylene), Newman Crop Spray 11E ${ }^{\mathrm{TM}}$ (aceite paraffinic), Nu-Film P (poly-1-p menthene) y Spray Gard (di-1-p menthene) en la reducción del daño por spray de sales en dos especies leñosas, encino siempre verde (Quercus ilex L.) y laurel (Prunus laurocerasus L.). Independientemente de la especie, los polímeros Nu-Film-P y Spay Gard no proporcionaron un grado significativo de protección contra el daño por sales, independiente de la concentración aplicada ( 1 o $2 \%)$, medida por las concentraciones de clorofila en la hoja, eficiencia fotosintética, necrosis foliar visual, contenido foliar de $\mathrm{Na}$ y $\mathrm{Cl}$ y crecimiento (altura, área foliar). El polímero formador de película Newman Crop Spray 11E $\mathrm{E}^{\mathrm{TM}}$ proporcionó solamente una semana de protección contra el daño por sales. El polímero Bond dio un significativo $(P<0.05)$ grado de protección contra la sal tres meses después de la aplicación, manifestado por más alto contenido de clorofila en la hoja, eficiencia fotosintética, altura y área foliar y más baja necrosis foliar visual y contenido de $\mathrm{Na}$ y $\mathrm{Cl}$, comparado con lo controles no tratados. En conclusión, los resultados indican que la aplicación de polímeros formadores de película da un grado significativo de protección hasta tres meses contra los daños de spray con sales en encino y laurel. Los resultados también indican que cuando las soluciones son aplicadas al 1 o $2 \%$ no hay problemas asociados con fitotoxicidad y degradación rápida en la superficie de la hoja. 